\title{
Severity and case fatality rates of COVID-19: A systematic review, meta-analysis and an exploratory meta-regression of risk factors
}

\author{
Chathurika Dhanasekara MBBS, PhD, Shao-Hua Chin PhD, Chanaka N. Kahathuduwa MBBS, PhD
}

\begin{abstract}
Background: We estimated the prevalence of severe or critical illness and case fatality of COVID-19 in a systematic review and meta-analysis and examined clinical, biochemical, and radiological risk factors in a meta-regression.

Methods: PRISMA guidelines were followed. PubMed, Scopus and Web of Science were searched using pre-specified keywords. Peer-reviewed empirical studies examining rates of severe illness, critical illness and case fatality among COVID-19 patients were analyzed. Randomeffects meta-analyses were performed and adjusted for publication bias. Meta-regression analyses examined the moderator effects of risk factors.

Results: The meta-analysis included 29 studies representing 2,090 individuals. Pooled prevalence rates of severe illness, critical illness and case fatality among COVID-19 patients were $15 \%, 5 \%$, and $0.8 \%$, respectively. There were significant heterogeneity and publication bias in these studies. Meta-regression analyses revealed that increasing age and elevated LDH consistently predicted severe / critical disease and case fatality. In addition, hypertension, fever and dyspnea at presentation, and elevated CRP predicted increased severity.

Conclusions: These predictors of severity and case fatality should allow clinicians to define at-risk endophenotypes. Differences in unadjusted vs. adjusted pooled estimates indicates limited utility of small-scale studies and underscores the importance of multinational studies to establish the morbidity and mortality rates in pandemics.
\end{abstract}

Keywords: SARS-CoV-2, COVID-19, case fatality, meta-analysis, meta-regression

\section{INTRODUCTION}

A novel corona virus, first identified in Wuhan, China, in late 2019, resulted in a pandemic by the first quarter of 2020; these widespread infections were attributed to the prolonged survival of the virus in the environment and extended length of preor post-symptomatic and potential asymptomatic shedding. ${ }^{1}$ While the virus is known to cause only a mild illness in a majority of cases, severe illness

Corresponding author: Chanaka Kahathuduwa Contact Information: Chanaka.Kahathuduwa@ttuhsc.edu DOI: 10.12746/swrccc.v9i41.921 characterized by respiratory distress requiring hospital admission is not uncommon. ${ }^{2}$ Furthermore, the virus has the potential to precipitate a life-threatening critical illness, characterized by respiratory failure, circulatory shock, and sepsis or other organ failure, requiring intensive care. ${ }^{3}$

An extensive body of literature published since the outset of the epidemic in China has examined the rates of severe and critically severe and the case fatality associated with COVID-19. However, the literature on COVID-19 has several limitations. First, due to lack of awareness and limited availability of training and resources to confirm the diagnosis, failure to recognize and code COVID-19 as the potential cause of morbidity and mortality may have contributed to 
under-estimation of the effects of COVID-19. ${ }^{4}$ In fact, a recent estimate suggested that approximately $86 \%$ cases of COVID-19 were not documented prior to January $23,2020 .{ }^{5}$ But screening only those who are at high risk may lead to over reporting of morbidity and mortality. Second, given that most datasets and publications are derived from retrospective chart review, as opposed to prospective methods, high measurement error is inevitable. ${ }^{6}$ Third, the literature on the outcomes mainly originates from tertiary care settings, distorting the overall clinical picture. ${ }^{7}$ Finally, including the same patients in multiple reports examining the same research question without clearly indicating this overlap is a major lapse in methodological and ethical standards. ${ }^{8}$

We conducted a systematic review of the available literature to identify publications with minimal potential overlap to estimate the prevalence of severe illness, critical illness, and case fatality among individuals with COVID-19 in random-effects meta-analyses to enhance generalizability. We adjusted our prevalence estimates by correcting for publication bias and underreporting. We also examined the effects of clinical, biochemical, and radiological risk factors moderating the between-study heterogeneity of the severity and case fatality rates.

\section{METHODS}

\section{SeARCH StRATEgY AND SELECTION CRITERIA}

All procedures were conducted in accord with the Preferred Reporting Items for Systematic Reviews and Meta-analyses (PRISMA) guidelines. PubMed, Scopus and Web of Science databases were searched on March 7, 2020, to identify studies that have been published in 2020 examining the prevalence of severe illness, critically severe illness, and mortality associated with COVID-19 infection using pre-determined keyword combinations (S1 Table). No language restrictions were applied. Duplicate records were removed, and titles and abstracts were screened for pre-defined eligibility criteria (Figure 1) by two independent raters (CD and CK or SC). Records published in Chinese were translated to American English using Google translator, and a native Chinese speaker with doctoral level training (SC) examined the original records in Chinese. Fulltext manuscripts of records that were considered eligible after the initial screening were re-examined. Two study personnel examined all eligible full-text manuscripts using the 9-item Quality Assessment Tool for Case Series Studies of the National Heart, Lung and Blood Institute.

\section{Data EXtRAction}

Data from the eligible manuscripts were organized into pre-defined data fields. The data fields included the total sample size and number of participants with severe illness, critically severe illness, and mortality. Severe illness was operationally defined as having either respiratory distress with $\mathrm{RR} \geq 30 / \mathrm{min}$, resting peripheral oxygen saturation of $\leq 93 \%$, or arterial partial pressure of oxygen $\leq 300 \mathrm{mmHg}$, or requiring hospitalization. Critical illness was defined as having respiratory failure, circulatory shock, end-organ failure, any combination of the above, or requiring intensive care. In addition, the following variables were extracted as potential covariates of the above outcomes. Central tendency (i.e., mean or median) and dispersion (i.e., SD, SE, $95 \% \mathrm{CI}, \mathrm{IQR}$ or range) of age were extracted. When not reported, study level means and standard deviations for age were imputed from the available statistics (i.e., median, IQR or range). ${ }^{9}$ Proportions of the following variables within a study sample were extracted: age $\leq 18$ years, age $\geq 60$ years, female sex, diabetes mellitus, hypertension, heart disease, chronic liver disease, chronic kidney disease, chronic obstructive pulmonary disease, malignancy, immunosuppression (e.g., HIV), smoking and pregnancy. Proportions of patients with specific presenting symptoms (i.e., fever, cough, sore throat, shortness of breath, headache, diarrhea), asymptomatic cases, specific laboratory parameters (i.e., positive nucleic acid test for COVID-19, leukopenia, leukocytosis, thrombocytopenia, lymphopenia, elevated lactate dehydrogenase (LDH), elevated C-reactive protein (CRP), elevated erythrocyte sedimentation rate (ESR), high procalcitonin and high $\mathrm{D}$-dimer based on reference ranges considered in each study) and radiographic features (i.e., no 


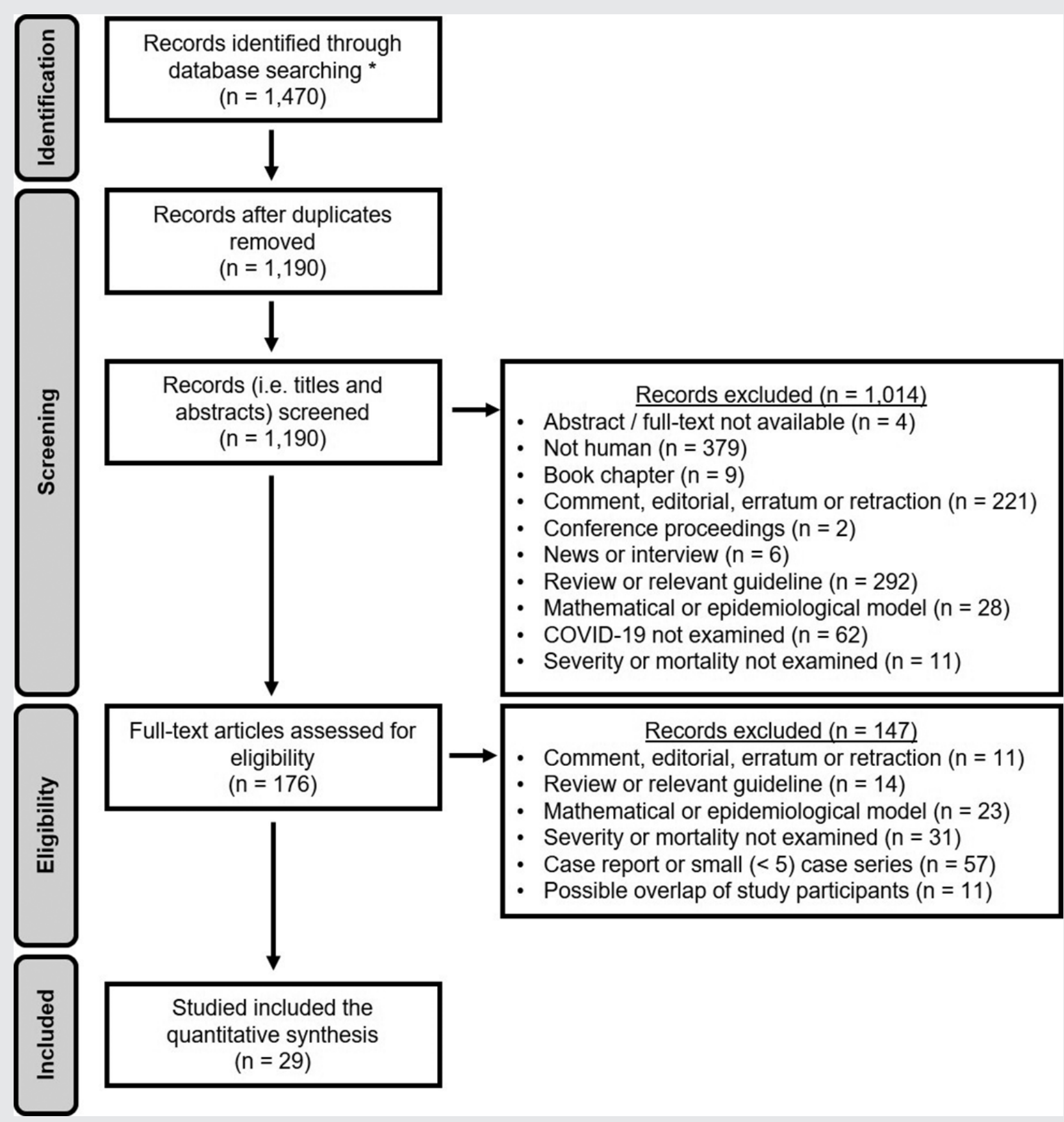

Figure 1. PRISMA flow chart outlining eligibility criteria and study selection.

lesions on computed tomography, patchy consolidation, ground glass opacities, peripheral distribution, and bilateral lung involvement or involvement of $\geq 3$ lobes).

\section{Data ANALYSIS}

Three separate DerSimonian-Laird randomeffects meta-analyses were performed using the 'meta' 
package (version 4.11-0) in R statistical software (version 3.6.2) to examine three primary outcomes: the prevalence of a) combined severe or critical COVID19 infection, b) critically severe COVID-19 infection, and c) COVID-19-associated mortality. ${ }^{10}$ Studies with both zero or $100 \%$ proportions were not excluded to ensure incorporation of all available data, which is known to ensure analytic consistency and minimize bias. ${ }^{11}$ Consistency of the findings of the meta-analyses were confirmed by leave-one-out sensitivity analyses. ${ }^{12}$ Given that under-reporting and publication bias could result in biased (i.e., smaller) prevalence estimates, publication bias was examined using funnel plots. Funnel plot asymmetry was confirmed using the traditional Egger tests and the Peters test, which is robust to small-study effects (i.e., biases caused by the likelihood of studies with extreme results despite small sample sizes getting published) and seems to perform better when using proportions or log odds as outcomes. ${ }^{13,14}$ The effect-sizes were imputed for estimated missing (i.e., unpublished / unreported) studies via the trim-and-fill method. ${ }^{15}$ The trim-and-fill method relies heavily on the assumption that the funnel plot of a meta-analysis should be symmetric and corrects for asymmetric distributions of effect-sizes by removing smaller studies that may contribute to asymmetric funnel plots, estimating a center of effect-sizes using the remaining effect-sizes and imputing effect-sizes until the funnel plot becomes symmetric. The meta-analyses were repeated including the effect-sizes of potentially missing studies imputed using the trim-and-fill method for each primary outcome. ${ }^{15}$ Heterogeneity of effect-sizes was quantified by calculating the Higgins' $I^{2}$ statistic for each meta-analysis. ${ }^{16}$ To explain the heterogeneity of the studies, ${ }^{17}$ exploratory univariate random-effects meta-regression analyses were performed to examine the moderator effects of each of the covariates described above.

\section{RESULTS}

Results of database search, subsequent screening, and eligibility assessment are summarized in a PRISMA flow diagram (Figure 1). Out of 2,564 records identified in the initial database search, 29 studies including data for 2,090 patients with COVID-19 were considered eligible. The proportions of females in the study samples ranged from $27.59 \%$ to $100.00 \%$. The mean age of the participants included in the studies ranged from 2 to 66 years. Four studies entirely recruited children, and one study exclusively recruited pregnant women. The included studies and their quality ratings are summarized in Table 1 . The summary statistics of all covariates are summarized in S2 Table.

Pooled prevalence of severe or critically severe illness among individuals with COVID-19 infection was estimated to be $14.6 \%(95 \% \mathrm{Cl}, 8.9 \%-23.1 \%)$ in the random-effects meta-analysis (Figure 2a). Excluding any single study from the meta-analysis (i.e., leaveone-out sensitivity analyses) did not significantly change the pooled prevalence estimate. However, the funnel plot of the effect-sizes was severely asymmetric, suggesting underreporting or publication bias (Figure 2b). Egger test indicated asymmetry $(t=-2.208, p=0.037)$, yet the Peters test was not significant, indicating that the funnel plot asymmetry may be driven by the small-study effect $(t=1.874$, $p=0.073)$. Seven effect-sizes were imputed to correct for the publication bias. When the random-effects meta-analysis was performed including these imputed effect-sizes (i.e., after correcting for publication bias), the prevalence of severe or critical illness increased to $25.8 \%(95 \% \mathrm{Cl}, 17.2 \%-36.8 \%)$ (Figure $2 \mathrm{c}$ ).

Significant heterogeneity was observed among the prevalence estimates of severe illness $\left(\tau^{2}=1.679\right.$; $\left.1^{2}=94 \%, p<0.001\right)$. Correcting for publication bias decreased this heterogeneity $\left(\mathrm{I}^{2}=85 \%, 95 \% \mathrm{Cl}\right.$, $80 \%-89 \%$ ); however, heterogeneity remained significant $(p<0.001)$. Exploratory univariate random-effects meta-regression analyses conducted with the aim of explaining the heterogeneity using the moderator effects of the considered covariates suggested that each of increasing mean age $(p=0.006)$ and prevalence of age $\geq 60$ years $(p<0.001)$, hypertension $(p<0.001)$, chronic kidney disease $(p=0.038)$, malignancy $(p=0.023)$, and chronic obstructive pulmonary disease $(p=0.025)$ were associated with a greater risk of severe or critical illness associated with COVID-19, while the prevalence of age $\leq 18$ years 
Table 1. Studies Meeting Eligibility Criteria

\begin{tabular}{|c|c|c|c|c|c|c|c|c|}
\hline \multirow[b]{2}{*}{ Study* } & \multirow[b]{2}{*}{\begin{tabular}{|l} 
Study \\
Setting
\end{tabular}} & \multirow[b]{2}{*}{ Study Period } & \multirow[b]{2}{*}{$\begin{array}{l}\text { Sample } \\
\text { Size }\end{array}$} & \multirow{2}{*}{$\begin{array}{l}\text { Severe / } \\
\text { Critical } \\
\text { Illness }\end{array}$} & \multirow[b]{2}{*}{$\begin{array}{l}\text { Critical } \\
\text { Illness }\end{array}$} & \multirow[b]{2}{*}{ Mortality } & \multicolumn{2}{|c|}{ Quality $\dagger$} \\
\hline & & & & & & & Rater 1 & Rater 2 \\
\hline Cai (24) & In-patient $^{1}$ & Jan19-Feb03 & 10 & 0 & 0 & 0 & Good & Good \\
\hline Chen C (25) & Out patient & Jan-Feb & 150 & NA & 24 & 11 & Good & Good \\
\hline Chen H (26) & In-patient $^{2}$ & Jan20-Jan31 & 9 & 0 & 0 & 0 & Good & Good \\
\hline Chen L (27) & In-patient & Jan14-Jan29 & 29 & 14 & 5 & 2 & Good & Good \\
\hline Chen N (28) & In-patient & Jan01-Jan20 & 99 & 33 & 23 & 11 & Good & Good \\
\hline Chen W (29) & In-patient & NA & 57 & 18 & $\mathrm{NA}$ & NA & Fair & Poor \\
\hline Feng (30) & In-patient $^{1}$ & Jan16-Feb06 & 15 & 0 & 0 & 0 & Fair & Good \\
\hline Li K (31) & In-patient & Jan-Feb & 83 & 25 & 0 & NA & Good & Good \\
\hline Li Y (32) & In-patient & Jan-Feb & 54 & 14 & NA & 0 & Good & Good \\
\hline Liu C (33) & In-patient & Jan23-Feb08 & 32 & 4 & NA & 0 & Fair & Fair \\
\hline Liu K (34) & In-patient & Dec30-Jan24 & 137 & 34 & 26 & 16 & Good & Good \\
\hline Liu W (35) & In-patient & Dec30-Jan15 & 78 & 20 & 8 & 2 & Good & Good \\
\hline Liu Y (36) & In-patient & Jan11-Jan20 & 12 & 9 & 3 & 0 & Good & Good \\
\hline Tian (37) & Emergency & Jan20-Feb10 & 262 & 46 & 0 & 3 & Good & Good \\
\hline Wang D (a) (38) & In-patient $^{1}$ & Jan25-Feb21 & 31 & 0 & 0 & 0 & Fair & Good \\
\hline Wang D (b) (39) & In-patient & Jan01-Jan28 & 138 & 36 & 36 & 6 & Good & Good \\
\hline Wang L (40) & In-patient & Jan21-Feb05 & 18 & NA & 2 & 0 & Good & Good \\
\hline Wu J (41) & In-patient & Jan22-Feb14 & 80 & 3 & 3 & 0 & Good & Good \\
\hline $\mathrm{Wu} \mathrm{W}(42)$ & Out patient & Jan19-Jan25 & 40 & 17 & 4 & 2 & Good & Good \\
\hline Xia W (43) & In-patient $^{1}$ & Jan23-Feb8 & 20 & 1 & 0 & 0 & Fair & Good \\
\hline Xu X (44) & $\begin{array}{l}\text { Public health } \\
\text { data }\end{array}$ & Jan10-Jan26 & 62 & 1 & 1 & 0 & Good & Good \\
\hline $\mathrm{Xu} \mathrm{Y} \mathrm{(45)}$ & In-patient & Jan-Feb & 50 & 13 & 3 & 0 & Good & Good \\
\hline Yang (3) & In-patient & Jan17-Feb10 & 149 & 23 & 23 & 0 & Good & Good \\
\hline Yao (46) & $\begin{array}{l}\text { Public health } \\
\text { data }\end{array}$ & Jan01-Feb07 & 195 & 5 & 5 & 0 & Fair & Good \\
\hline Young (47) & $\begin{array}{l}\text { Public health } \\
\text { data }\end{array}$ & Jan22-Jan31 & 18 & 6 & 2 & 0 & Good & Good \\
\hline Zhang J (48) & In-patient & Jan16-Feb03 & 140 & 58 & NA & NA & Good & Good \\
\hline Zhang M (49) & In-patient & Jan18-Feb03 & 9 & 0 & 0 & 0 & Good & Good \\
\hline Zhao (50) & In-patient & NA & 101 & 14 & NA & NA & Good & Fair \\
\hline Zhu (51) & In-patient & Dec01-Feb15 & 12 & 0 & 0 & 0 & Good & Good \\
\hline
\end{tabular}

* All studies were published in year 2020. Young et al. (2020) was based on data collected in Singapore. All the studies were based on data collected in China.

$\dagger$ Quality of studies were examined using the National Heart, Lung and Blood Institute's 9-item Quality Assessment Tool for Case Series Studies.

${ }^{1}$ Study sample was made-up exclusively of children.

${ }^{2}$ Study sample was made-up exclusively of pregnant women. 


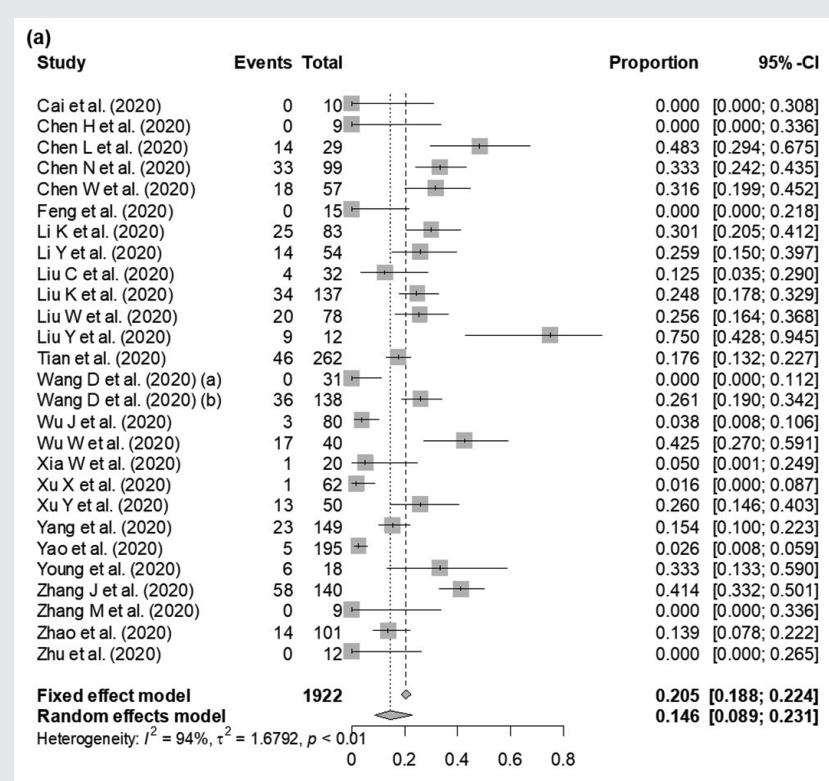

(b)

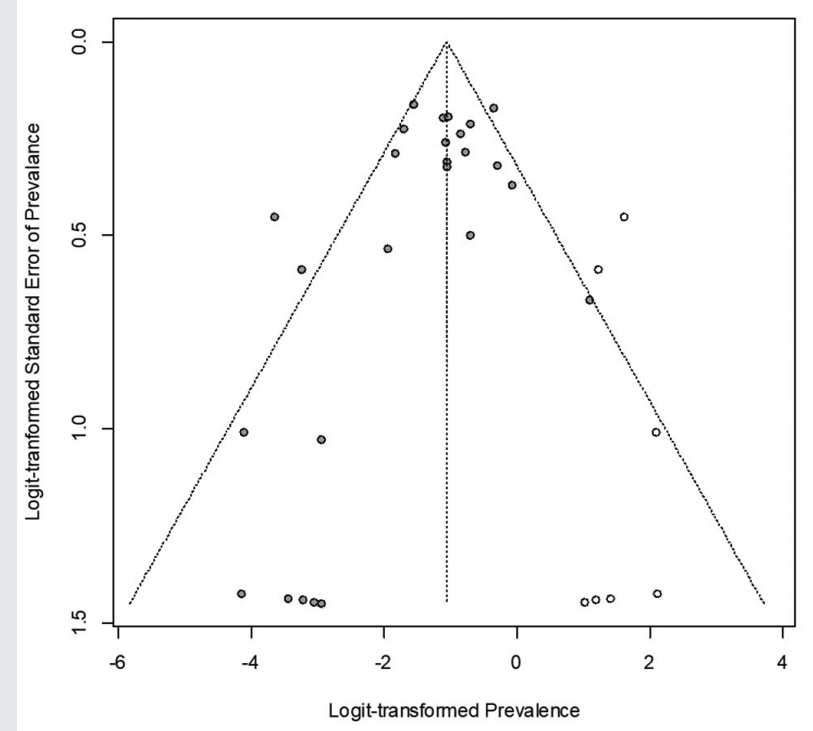

(c)

Random effects model Heterogeneity: $I^{2}=85 \%, \tau^{2}=1.7802, p<0.01$

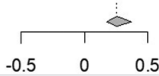

$0.258[0.172 ; 0.368]$

Figure 2. Results of random-effects meta-analysis examining the pooled prevalence of combined severe and critical illness among individuals with COVID-19. a) Forest plot; b) Funnel plot depicting publication bias and imputed effect-sizes to correct for publication bias; c) Results corrected for publication bias. $(p=0.007)$ within a sample was associated with a reduced risk of severe or critical illness. The prevalence of the presenting clinical features of fever $(p<0.001)$, dyspnea $(p=0.028)$, and diarrhea ( $p=0.026)$; laboratory findings of lymphocytopenia $(p=0.003)$, elevated LDH $(p<0.001)$, CRP $(p<0.001)$, and D-dimer levels $(p<0.001)$; and bilateral lung involvement or involvement of $\geq 3$ lung lobes $(p=0.006)$ was associated with increased risk of severe or critically severe illness, while having no radiological features on chest CT was associated with decreased risk of severe illness $(p=0.003)$. The results of all univariate meta-regression analyses examining the moderator effects of the covariates on the prevalence of severe or critical illness in COVID19 infection and their effects on heterogeneity are summarized in S3 Table.

The random-effects meta-regression analyses revealed a pooled estimate of $4.8 \%(95 \% \mathrm{Cl}, 2.4 \%-$ $9.5 \%)$ for the prevalence of critical illness in COVID-19 infection (Figure 3a). Leave-one-out meta-regression analyses did not significantly change this estimate. The funnel plot (Figure $3 b$ ) was suggestive of underreporting or publication bias. This was confirmed in an Egger test $(t=-5.099, p<0.001)$, yet the Peters test was not significant $(t=-0.319$, $p=0.758$ ). Eleven effect-sizes had to be imputed to statistically correct for this bias and after correction, the pooled prevalence of critical illness in COVID-19 infection increased to $16.3 \%(95 \% \mathrm{Cl}, 9.8 \%-25.7 \%)$ (Figure 3c).

Significant heterogeneity of effect-sizes was also a concern for the meta-analysis of prevalence of critical illness $\left(\tau^{2}=1.994 ; I^{2}=92 \%, p<0.001\right)$. Correcting for publication bias decreased this heterogeneity $\left(\mathrm{I}^{2}=78 \%, 95 \% \mathrm{Cl}, 69 \%-84 \%\right)$; however, heterogeneity remained significant $(p<0.001)$. Univariate metaregression analyses suggested increased risk of critical illness associated with sample characteristics of increasing mean age $(p=0.002)$, prevalence of age $\geq 60$ years $(p<0.001)$, comorbid hypertension $(p<0.01)$, cardiac disease $(p=0.023)$, and malignancy $(p=0.041)$. Similarly, the prevalence of fever $(p=0.044)$, dyspnea $(p=0.042)$, and fatigue $(p=0.036)$ on presentation; the prevalence of increased LDH $(p=0.003)$, CRP 


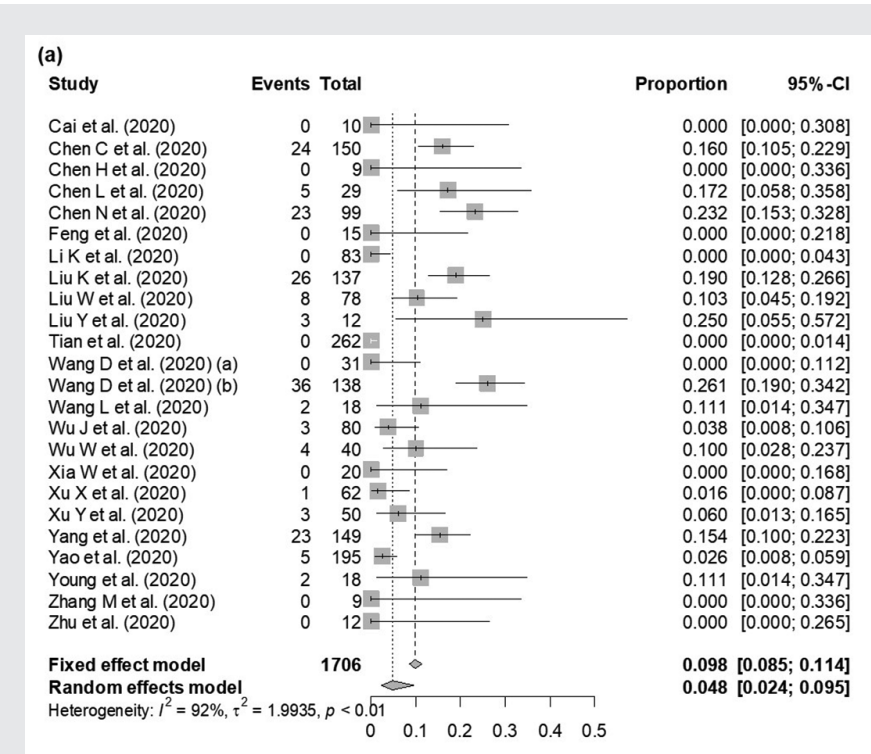

(b)

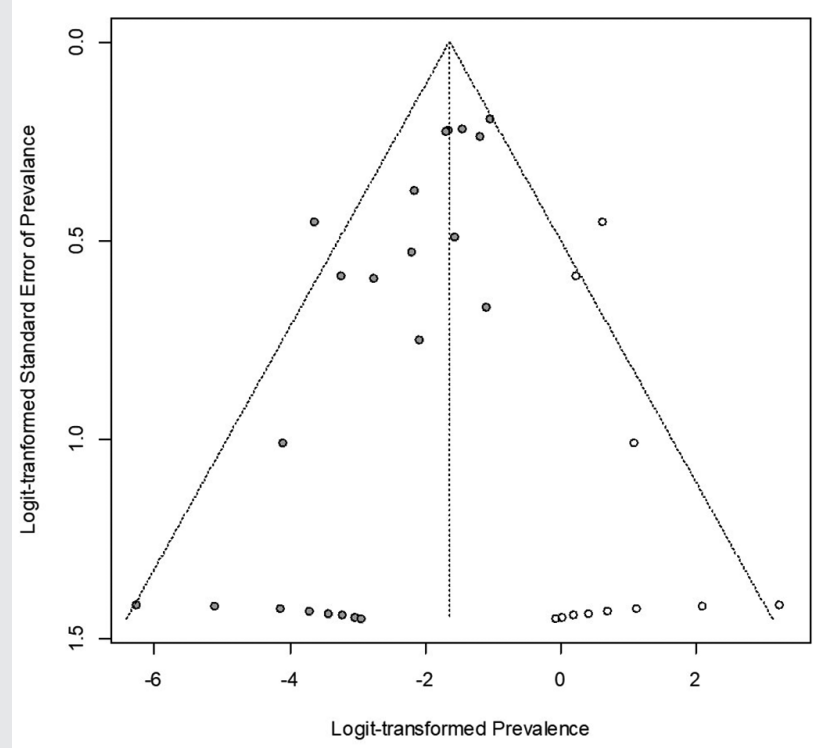

(c)

Random effects model
Heterogeneity. $I^{2}=78 \%, \tau^{2}=2.1129, p<0.01$

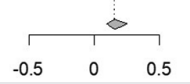

$0.163[0.098 ; 0.257]$

Figure 3. Results of random-effects meta-analysis examining the pooled prevalence of critical illness among individuals with COVID-19. a) Forest plot; b) Funnel plot depicting publication bias and imputed effect-sizes to correct for publication bias; c) Results corrected for publication bias. $(p=0.008)$, and D-dimer $(p=0.021)$ were associated with a greater risk of critical illness (S4 Table).

Prevalence of COVID-19 associated mortality was $0.8 \%(95 \% \mathrm{Cl}, 0.2 \%-2.9 \%)$ based on the randomeffects meta-analysis (Figure 4a), and this estimate was minimally affected by leave-one-out sensitivity analyses. However, as with severe and critically severe illness, publication bias and potential underreporting were apparent based on significant funnel plot asymmetry $(t=-5.203, p<0.001)$ (Figure $4 b)$. A Peters test was not significant and suggested the high likelihood of the funnel asymmetry being driven by small-study effect $(t=0.097, p=0.924)$. Trim-and-fill method, which corrects for both publication bias and small-study effect, imputed 13 effect-sizes to account for missing / unreported effects in an attempt to statistically correct for the publication bias. Conducting the meta-regression analyses with the addition of these effect-sizes revealed a pooled estimate of $7.4 \%(95 \%$ $\mathrm{Cl}, 4.5 \%-11.9 \%)$ for the mortality rate associated with COVID-19 infection (Figure 4c).

Heterogeneity of effect-sizes on prevalence of mortality in COVID-19 was also a concern $\left(\tau^{2}=2.996\right.$; $\left.I^{2}=86 \%, p<0.001\right)$. Correction for publication bias decreased the heterogeneity $\left(\mathrm{I}^{2}=61 \%, 95 \% \mathrm{Cl}\right.$, $45 \%-73 \%)$, yet the heterogeneity remained significant $(p<0.001)$. Univariate meta-regression analyses modeling heterogeneity indicated increased mortality risks associated with increasing mean age $(p<0.001)$, prevalence of age $\geq 60$ years $(p=0.011)$, presenting with fatigue $(p=0.048)$, leukocytosis $(p=0.007)$, high LDH $(p=0.030)$, and low albu$\min (p<0.001)$. The prevalence of age $\leq 18$ years $(p=0.036)$ was associated with a decreased risk of COVID-19-associated mortality (S5 Table).

\section{Discussion}

In this systematic review and meta-analysis, we comprehensively and systematically examined the available literature to estimate the prevalence of morbidity and mortality associated with SRAS-CoV-2 infection. Despite the large number of articles that were reviewed, our quantitative synthesis was limited to 29 studies representing data from 2,090 individuals. 


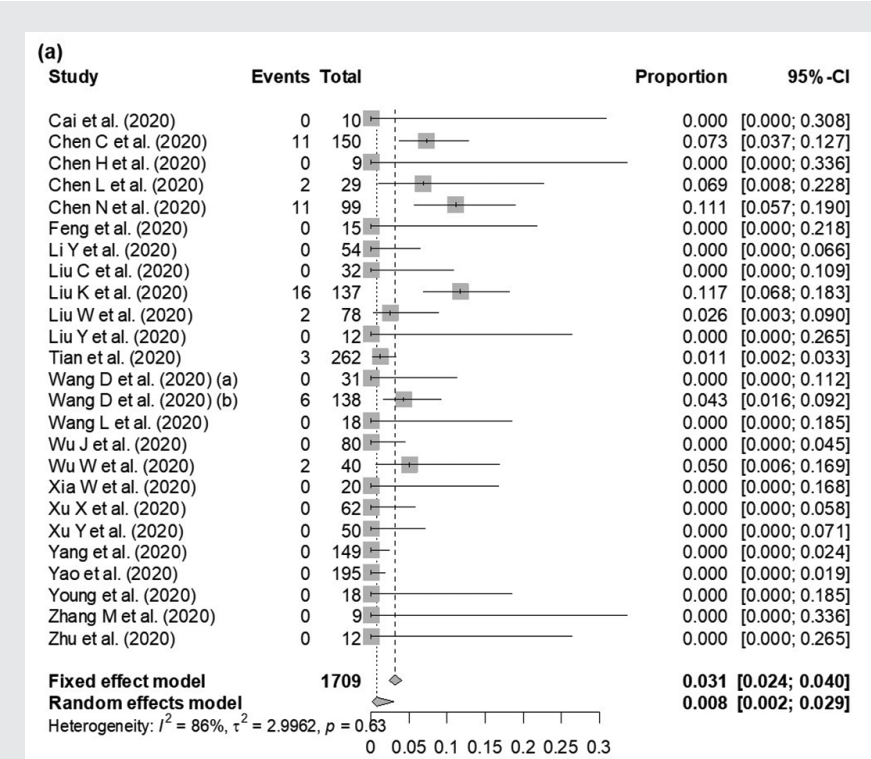

(b)

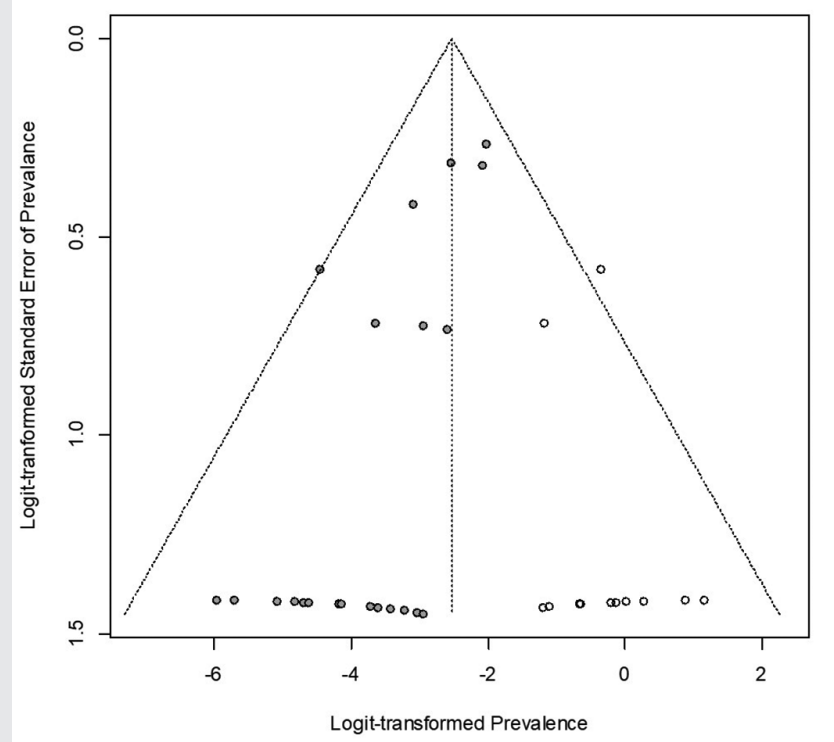

(c)

Random effects model

Reterogeneity: $I^{2}=61 \%, \tau^{2}=1.4060, p<0.01$
Hen

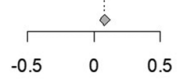

$0.074[0.045 ; 0.119]$

Figure 4. Results of random-effects meta-analysis examining the pooled case fatality rate among individuals with COVID-19. a) Forest plot; b) Funnel plot depicting publication bias and imputed effect-sizes to correct for publication bias; c) Results corrected for publication bias.
Our literature-based estimates of severe illness, critical illness, and case fatality rates among patients with COVID-19 were $15 \%, 5 \%$, and $0.8 \%$, respectively. After adjusting for underreporting and publication bias, COVID-19-associated prevalence of severe illness, critical illness and case fatality increased to $26 \%, 16 \%$, and $7.4 \%$, respectively.

Our unadjusted random-effects estimates of severe illness requiring hospitalization (15\%) and critical illness requiring intensive care admission $(5 \%)$ are consistent with the estimates of COVID-19associated morbidity based on large individual-level datasets. ${ }^{18}$ Therefore, the unadjusted findings of our meta-analysis regarding severity of illness corroborate the inferences made based on current surveillance systems. However, the unadjusted mortality rate observed in our analysis $(0.8 \%, 95 \% \mathrm{Cl}, 0.2 \%-$ $2.9 \%$ ) is lower than the COVID-19-associated mortality rates in China $(3.6 \%)$ or globally $(3.4 \%)$ at the end of February 2020 (i.e., the time represented in the reviewed publications).

Retrospective patient data and the literature derived from such data could be systematically biased toward both overestimating and / or underestimating morbidity and mortality. The reviewed studies largely represent tertiary care settings, of which the capacity may have been overridden minimally, if at all, despite the high reproductive number $\left(R_{0}\right)$ at the time of sampling. As such, the outcomes of our unadjusted random-effects meta-analyses, which accounts for the random variability of effects between studies, can be inferred to be a generalizable representation of morbidity and mortality rates applicable to well-trained and equipped healthcare settings in which resources are not overwhelmed. This estimate, therefore, represents COVID-19 associated mortality in regions with a low $R_{0}$.

However, reverse-causation bias caused by failing to capture the deaths that may not reach healthcare facilities / be diagnosed prior to death could contribute to underestimation. ${ }^{19}$ The effect-sizes imputed to correct for publication bias may, in fact, represent severity and case fatality rates of settings in which the demand exceeds the available resources. ${ }^{20}$ While optimized utilization of healthcare facilities by 
maintaining a low $\mathrm{R}_{0}$ may reduce the mortality rates to as low as $0.8 \%$, overwhelming healthcare resources may increase the overall case fatality rate to $7.4 \%$, or even greater as represented by the effect-sizes we have imputed. ${ }^{21}$

In our meta-regression analyses that examined risk factors, increasing age and age $\geq 60$ years consistently stood out as a risk factor, while age $\leq 18$ years consistently remained a protective factor, being consistent with the current literature. Angiotensin converting enzyme (ACE)-2 receptors are generally up regulated in patients with hypertension or heart failure who receive ACE-inhibitors and angiotensin-II receptor blockers. ${ }^{22}$ COVID-19 enters cells by binding to ACE-2 receptors, increasing their risk of infection and development of severe clinical illness. ${ }^{22}$ Consistently, we observed an increased risk of severity with hypertension and cardiovascular disease. Presenting with fever emerged as a risk factor for severe and critical illness and mortality, underscoring the importance of body temperature as a screening tool.

This systematic review and meta-analysis was conducted at the outset of the COVID-19 pandemic in an effort to determine morbidity and mortality rates associated with the disease. At the time the literature was reviewed, almost all the studies reporting morbidity and mortality data originated in China. Thus, the generalizability of the findings of this study are limited. In addition, our systematic review has some methodological limitations. First, while we eliminated studies with overlapping samples by screening for overlaps of institutes, study dates, and authors, we cannot be $100 \%$ certain of exclusion of all overlapping data. Second, a high degree of heterogeneity was a concern. However, this should be expected in any meta-analysis due to the variability in methodology and study samples. This limitation was mitigated partially by conducting random-effects meta-analyses to pool the estimates. More important, we used heterogeneity to explore the risk factors in meta-regression analyses. ${ }^{16}$ Third, the reviewed literature contained hardly any information regarding the types of medications used by patients (e.g., ACE inhibitors, angiotensin II receptor blockers), anthropometric parameters (e.g., BMI, fat mass) or the dietary patterns of the patients. Furthermore, the reviewed articles lacked data on specific treatment protocols or specific medications used for management of COVID-19. As such, the contribution of these moderator effects of these important variables could not be evaluated. Fourth, the protocol was not pre-registered. Finally, funnel plots of all three meta-analyses were visually asymmetric and the statistical tests indicated that these asymmetries might be driven mostly by small-study effects. While the trim-and-fill analysis can correct for asymmetries in funnel plots due to both small-study effect and underreporting, the analysis strongly assumes the symmetry of funnel plot, ${ }^{15}$ which may not hold true in the absence of publication bias. Furthermore, the accuracy of the estimates of trim-and-fill analysis is questionable in the presence of between-study heterogeneity, and the imputed data may contribute to artificially decrease the uncertainty of the pooled estimates. ${ }^{23}$ Thus, we emphasize the need to exercise caution when interpreting the findings that have been corrected for publication bias using the trim-and-fill analyses.

\section{Conclusions}

In conclusion, COVID-19 associated overall rates of hospitalization, intensive care, and case fatality were estimated to be $15 \%, 5 \%$, and $0.8 \%$, respectively, in our meta-analysis. Adjusting for potential underreporting and publication bias increased these estimates to $26 \%, 16 \%$, and $7.4 \%$, respectively, yet caution is advised in interpreting these adjusted outcomes due to limitations associated with the statistical methods used for adjustment. These differences in unadjusted vs. adjusted pooled estimates and the high between study heterogeneity indicate the limited generalizability of prevalence estimates derived from small-scale studies and underscore the importance of relying on multinational studies to establish the morbidity and mortality rates in pandemics (e.g., COVID19). Hypertension, fever and dyspnea at presentation, and elevated CRP seem to predict increased disease severity, while increasing age and elevated LDH seem to consistently predict severity and case fatality. These risk factors should inform clinicians to define endophenotypes possessing a greater risk. 


\section{Supporting information- Supplementary tables}

S1 Table. Keywords and keyword combinations used to screen the PubMed, Scopus, and Web of Science electronic databases.

S2 Table. Summary statistics of all covariates used in univariate meta-regression analyses.

S3 Table. Results of all univariate meta-regression analyses examining the moderator effects of covariates on the prevalence of combined severe or critical illness in COVID-19.

S4 Table. Results of all univariate meta-regression analyses examining the moderator effects of covariates on the prevalence critical illness in COVID-19.

S5 Table. Results of all univariate meta-regression analyses examining the moderator effects of covariates on the case-fatality rate in COVID-19.

Article citation: Dhanasekara C, Chin S-H, Kahathuduwa $\mathrm{CN}$. Severity and case fatality rates of COVID-19: a systematic review, meta-analysis and an exploratory meta-regression of risk factors. The Southwest Respiratory and Critical Care Chronicles 2021;9(41):8-19

From: Department of Psychiatry (CNK), Department of Laboratory Science and Primary Care (CNK), Department of Surgery (CSD), Texas Tech University Health Sciences Center, Lubbock, Texas; Medical Science Department (SHC), Protech Pharmaservices Corporation, Taipei City, Taiwan

Submitted: $8 / 6 / 2021$

Accepted: 10/9/2021

Reviewer: Jeff Dennis PhD

Conflicts of interest: none

This work is licensed under a Creative Commons

Attribution-ShareAlike 4.0 International License.

\section{REFERENCES}

1. Huang C, Wang Y, Li X, et al. Clinical features of patients infected with 2019 novel coronavirus in Wuhan, China. Lancet 2020;395(10223):497-506.

2. $\mathrm{Wu} \mathrm{Z}$, McGoogan JM. Characteristics of and important lessons from the Coronavirus Disease 2019 (COVID-19) Outbreak in China: Summary of a report of 72314 cases from the Chinese Center for Disease Control and Prevention. JAMA 2020;323(13):1239-1242.

3. Yang $\mathrm{X}, \mathrm{Yu} \mathrm{Y}, \mathrm{Xu}$ J, et al. Clinical course and outcomes of critically ill patients with SARS-CoV-2 pneumonia in Wuhan, China: a single-centered, retrospective, observational study. Lancet Respir Med 2020;8(5):475-481.

4. Lau H, Khosrawipour V, Kocbach $P$, et al. Internationally lost COVID-19 cases. J Microbiology, Immunology and Infection 2020;53(3):454-458.

5. Li R, Pei S, Chen B, et al. Substantial undocumented infection facilitates the rapid dissemination of novel coronavirus (SARS-CoV2). Science 2020;368(6490):489-493.

6. Matt V, Matthew $H$. The retrospective chart review: important methodological considerations. J educational evaluation for health professions 2013;10:12.

7. Delgado-Rodríguez M, Llorca J. Bias. J Epidemiol Community Health. 2004;58(8):635-41.

8. Bauchner H, Golub RM, Zylke J. Editorial concern-possible reporting of the same patients with COVID-19 in different reports. JAMA 2020;323(13):1256.

9. Wan X, Wang W, Liu J, Tong T. Estimating the sample mean and standard deviation from the sample size, median, range and/or interquartile range. BMC Med Res Methodol 2014; $14: 135$.

10. DerSimonian R, Laird N. Meta-analysis in clinical trials. Controlled clinical trials. 1986;7(3):177-88.

11. Friedrich JO, Adhikari NK, Beyene J. Inclusion of zero total event trials in meta-analyses maintains analytic consistency and incorporates all available data. BMC Med Res Methodol 2007; 7:5.

12. Patsopoulos NA, Evangelou E, Ioannidis JP. Sensitivity of between-study heterogeneity in meta-analysis: proposed metrics and empirical evaluation. Int J Epidemiol 2008;37(5): $1148-57$.

13. Egger M, Davey Smith G, Schneider M, et al. Bias in meta-analysis detected by a simple, graphical test. BMJ 1997; 315(7109):629-34.

14. Peters JL, Sutton AJ, Jones DR, et al. Comparison of two methods to detect publication bias in meta-analysis. JAMA 2006;295(6):676-80.

15. Duval S, Tweedie R. A nonparametric "trim and fill" method of accounting for publication bias in meta-analysis. Journal of the American Statistical Association 2000;95(449): 89-98.

16. Higgins J, Thompson SG. Quantifying heterogeneity in a meta analysis. Statistics in medicine 2002;21(11):1539-58.

17. Thompson SG, Sharp SJ. Explaining heterogeneity in meta analysis: a comparison of methods. Statistics in medicine 1999; 18(20):2693-708.

18. World Health Organization. Clinical management of severe acute respiratory infection (SARI) when COVID-19 disease is 
suspected: interim guidance. https:/www.who.int/docs/defaultsource/coronaviruse/clinical-management-of-novel-cov.pdf Published March 13, 2020. Accessed October 7, 2021.

19. Lipsitch M, Donnelly CA, Fraser C, et al. Potential biases in estimating absolute and relative case-fatality risks during outbreaks. PLoS Negl Trop Dis 2015;9(7):e0003846.

20. Ji Y, Ma Z, Peppelenbosch MP, et al. Potential association between COVID-19 mortality and health-care resource availability. Lancet Glob Health 2020;8(4):e480.

21. Baud D, Qi X, Nielsen-Saines K, et al. Real estimates of mortality following COVID-19 infection. Lancet Infect Dis 2020;20(7):773.

22. Fang L, Karakiulakis G, Roth M. Are patients with hypertension and diabetes mellitus at increased risk for COVID-19 infection? Lancet Respir Med 2020;8(4):e21.

23. Terrin N, Schmid CH, Lau J, Olkin I. Adjusting for publication bias in the presence of heterogeneity. Statistics in medicine 2003;22(13):2113-26.

24. Cai J, Xu J, Lin D, et al. A Case Series of children with 2019 novel coronavirus infection: clinical and epidemiological features. Clin Infect Dis 2020;71(6):1547-1551.

25. Chen C, Yan JT, Zhou N, et al. [Analysis of myocardial injury in patients with COVID-19 and association between concomitant cardiovascular diseases and severity of COVID-19]. Zhonghua Xin Xue Guan Bing Za Zhi. 2020;48(0):E008.

26. Chen $\mathrm{H}$, Guo J, Wang $\mathrm{C}$, et al. Clinical characteristics and intrauterine vertical transmission potential of COVID-19 infection in nine pregnant women: a retrospective review of medical records. Lancet 2020;395(10226):809-15.

27. Chen L, Liu HG, Liu W, et al. [Analysis of clinical features of 29 patients with 2019 novel coronavirus pneumonia]. Zhonghua Jie He He Hu Xi Za Zhi 2020;43(0):005.

28. Chen N, Zhou M, Dong X, et al. Epidemiological and clinical characteristics of 99 cases of 2019 novel coronavirus pneumonia in Wuhan, China: a descriptive study. Lancet 2020; 395(10223):507-13.

29. Chen W, Lan Y, Yuan X, et al. Detectable 2019-nCoV viral RNA in blood is a strong indicator for the further clinical severity. Emerg Microbes Infect 2020;9(1):469-73.

30. Feng K, Yun YX, Wang XF, et al. Analysis of CT features of 15 children with 2019 novel coronavirus infection. Chinese J Pediatrics. 2020;58:E007.

31. $\mathrm{Li} \mathrm{K}, \mathrm{Wu}$ J, Wu F, et al. The clinical and chest CT features associated with severe and critical COVID-19 Pneumonia. Invest Radiol 2020;55(6):327-331.

32. Li YY, Wang WN, Lei Y, et al. [Comparison of the clinical characteristics between RNA positive and negative patients clinically diagnosed with 2019 novel coronavirus pneumonia]. Zhonghua Jie He He Hu Xi Za Zhi 2020;43(0): E023.
33. Liu C, Jiang ZC, Shao CX, et al. [Preliminary study of the relationship between novel coronavirus pneumonia and liver function damage: a multicenter study]. Zhonghua Gan Zang Bing Za Zhi 2020;28(2):148-52.

34. Liu K, Fang YY, Deng Y, et al. Clinical characteristics of novel coronavirus cases in tertiary hospitals in Hubei Province. Chin Med J (Engl) 2020;133(9):1025-1031.

35. Liu W, Tao ZW, Lei W, et al. Analysis of factors associated with disease outcomes in hospitalized patients with 2019 novel coronavirus disease. Chin Med J (Engl) 2020; 133(9):1032-1038.

36. Liu Y, Yang $Y$, Zhang $C$, et al. Clinical and biochemical indexes from 2019-nCoV infected patients linked to viral loads and lung injury. Sci China Life Sci 2020;63(3):364-74.

37. Tian S, Hu N, Lou J, et al. Characteristics of COVID-19 infection in Beijing. J Infect 2020;80(4):401-406.

38. Wang D, Ju XL, Xie F, et al. [Clinical analysis of 31 cases of 2019 novel coronavirus infection in children from six provinces (autonomous region) of northern China]. Zhonghua Er Ke Za Zhi 2020;58(4):E011.

39. Wang D, Hu B, Hu C, et al. Clinical characteristics of 138 hospitalized patients with 2019 novel coronavirus-infected pneumonia in Wuhan, China. JAMA 2020;323(11):1061-1069.

40. Wang L, Gao YH, Lou LL, Zhang GJ. The clinical dynamics of 18 cases of COVID-19 outside of Wuhan, China. Eur Respir J 2020;55(4):2000398.

41. Wu J, Liu J, Zhao X, et al. Clinical characteristics of imported cases of COVID-19 in Jiangsu Province: a multicenter descriptive study. Clin Infect Dis 2020;71(15):706-712.

42. Wu WS, Li YG, Wei ZF, et al. [Investigation and analysis on characteristics of a cluster of COVID-19 associated with exposure in a department store in Tianjin]. Zhonghua Liu Xing Bing Xue Za Zhi 2020;41(4):489-93.

43. Xia W, Shao J, Guo Y, et al. Clinical and CT features in pediatric patients with COVID-19 infection: Different points from adults. Pediatr Pulmonol 2020;55(5):1169-1174.

44. $\mathrm{Xu} X W, \mathrm{Wu} X X$, Jiang $\mathrm{XG}$, et al. Clinical findings in a group of patients infected with the 2019 novel coronavirus (SARSCov-2) outside of Wuhan, China: retrospective case series. BMJ. 2020;368:m606.

45. Xu YH, Dong JH, An WM, et al. Clinical and computed tomographic imaging features of novel coronavirus pneumonia caused by SARS-CoV-2. J Infect 2020;80(4):394400 .

46. Yao Y, Tian Y, Zhou J, et al. Epidemiological characteristics of 2019-nCOV infections in Shaanxi, China by February 8, 2020. Eur Respir J 2020;55(4):2000310.

47. Young BE, Ong SWX, Kalimuddin S, et al. Epidemiologic features and clinical course of patients infected with SARSCoV-2 in Singapore. JAMA 2020;323(15):1488-1494. 
48. Zhang JJ, Dong X, Cao YY, et al. Clinical characteristics of 140 patients infected with SARS-CoV-2 in Wuhan, China. Allergy 2020;75(7):1730-1741.

49. Zhang MQ, Wang XH, Chen YL, et al. [Clinical features of 2019 novel coronavirus pneumonia in the early stage from a fever clinic in Beijing]. Zhonghua Jie He He Hu Xi Za Zhi 2020;43(0):E013.
50. Zhao W, Zhong Z, Xie X, et al. Relation between chest $\mathrm{CT}$ findings and clinical conditions of coronavirus disease (COVID-19) pneumonia: a multicenter study. AJR Am J Roentgenol 2020:1-6.

51. Zhu ZW, Tang JJ, Chai XP, et al. [Comparison of heart failure and 2019 novel coronavirus pneumonia in chest CT features and clinical characteristics]. Zhonghua Xin Xue Guan Bing Za Zh. 2020;48(0):E007. 